\title{
ANÁLISE BIBLIOGRÁFICA DA ASSISTÊNCIA DE ENFERMAGEM PRESTADA A MULHER VÍTIMA DE VIOLÊNCIA DOMÉSTICA
}

DOI: 10.48140/digitaleditora.2021.005.2

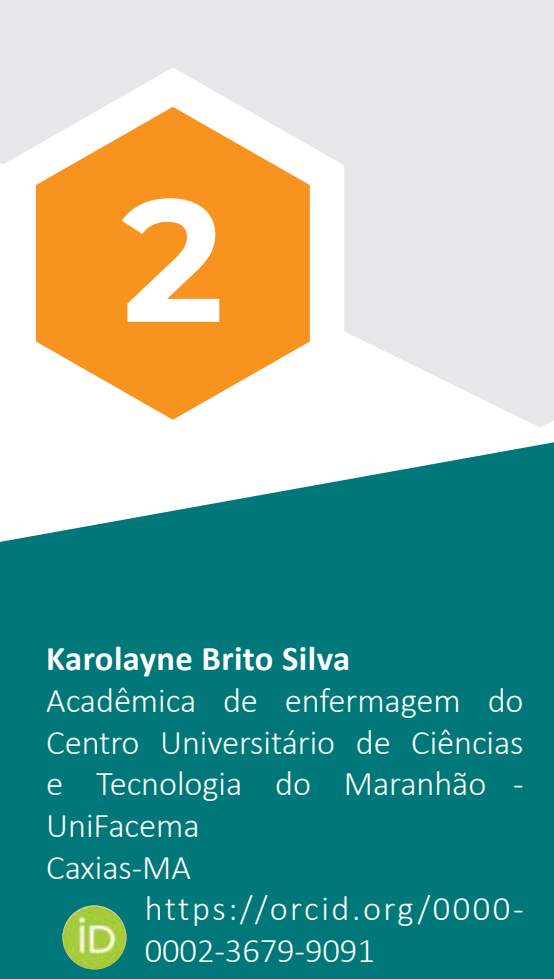

Maria Eduarda Leal de Carvalho Santos

Acadêmica de enfermagem do Centro Universitário de Ciências e Tecnologia do Maranhão UniFacema

Caxias-MA

(iD) $0003-3400-0570$

Pedro Henrique Medeiros de

\section{Andrade}

Acadêmico de enfermagem do Centro Universitário de Ciências e Tecnologia do Maranhão UniFacema
(iD) https://orcid.org/0000-
(iD) 0003-1819-9540

Najra Danny Pereira Lima

Mestre em Análise

Comportamento Aplicada

Centro Paradigma/ Docente do Centro Universitário de Ciências e Tecnologia do Maranhão UniFacema Caxias-MA

(iD) https://orcid.org/00000003-3673-0876

Maria Camila da Silva

Graduanda em Psicologia pelo Centro Universitário de Ciências e Tecnologia do MaranhãoUNIFACEMA

Caxias-MA

(iD) https://orcid.org/0000-

\section{Joyce de Sousa Leal}

Acadêmica de Enfermagem do Centro Universitário de Ciências e Tecnologia do Maranhão UniFacema Caxias-MA https://orcid.org/00000003-2972-016X
Simone Costa de Sousa Acadêmica de Enfermagem do Centro Universitário de Ciências e Tecnologia do Maranhão UniFacema Caxias-MA

(iD) https://orcid.org/0000 (D) 0003-4252-6548

Rondinelle dos Santos Chaves Enfermeiro Especialista em Preceptoria no SUS (Instituto Sírio Libanês de Ensino e Pesquisa, IEPSL)

São Paulo-SP

(iD) https://orcid.org/0000-

(iD) 0003-4941-8005/print

Mayanny da Silva Lima

Enfermeira Especialista em saúde pública/PSF pelo Instituto Athenas. Caxias-MA

(iD) http://orcid.org/00000002-6955-5675

Joerdanyelle Camilla Passos de Oliveira Rabelo

Enfermeira Especialista em Enfermagem do Trabalho- Instituto Athena

Caxias-MA

(iD) https://orcid.org/00000001-7997-9570 


\section{ANÁLISE BIBLIOGRÁFICA DA ASSISTÊNCIA DE ENFERMAGEM PRESTADA A MULHER VÍTIMA DE VIOLÊNCIA DOMÉSTICA}

DOI: $10.48140 /$ digitaleditora.2021.005.2

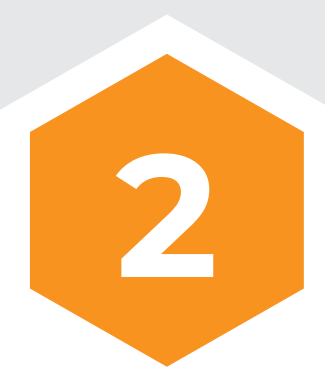

\section{RESUMO}

Objetivos: Objetivo: Descrever através da literatura vigente a assistência de enfermagem prestada à mulher vítima de violência doméstica.

Métodos: Este é um estudo bibliográfico do tipo revisão integrativa de literatura, de abordagem qualitativa que ocorreu nas bases de dados PubMed da National Library of Medicine e BVS (Biblioteca Virtual da Saúde), coordenada pela BIREME e composta de bases de dados bibliográficas produzidas pela Rede BVS, como LILACS, além da base de dados Medline e outros tipos de fontes de informação e CINAHL (Cumulative Index to Nursing and Allied Health Literature).

Resultados: Foram selecionadas 09 publicações completas para subsidiar resultados e discussão, incluíram-se apenas publicações de 2014 a 2020, no idioma português, inglês e espanhol. Os estudos abordavam sobre a assistência de enfermagem prestada à mulher vítima de violência doméstica, a realização das técnicas propedêuticas como inspeção, palpação e ausculta, como também a importância da abordagem holística para uma assistência de qualidade e humanizada

Conclusão: Desse modo, identifica - se as formas de assistência prestada pelos enfermeiros da atenção primária a mulher vítima de violência doméstica e a importância da realização da notificação, identificando assim as ações cabíveis, adversidades e possíveis intervenções que possa aperfeiçoar a assistência de enfermagem. 


\section{BIBLIOGRAPHIC ANALYSIS OF NURSING}

ASSISTANCE PROVIDED TO

\section{WOMAN VICTIM OF DOMESTIC VIOLENCE}

DOI: 10.48140/digitaleditora.2021.005.2

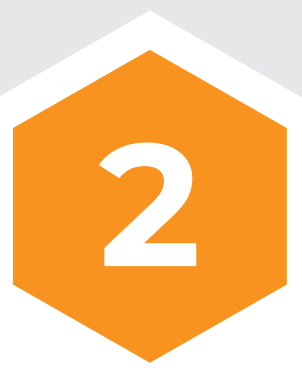

ABSTRACT

Recebido em: 30/11/2020

Aprovado em: 10/12/2020

Conflito de Interesse: não

Suporte Financeiro: não houve

Objectives: To describe through the current literature the nursing care provided to women victims of domestic violence.

Methods: This is a bibliographic study of the type integrative literature review, with a qualitative approach that occurred in the PubMed databases of the National Library of Medicine and VHL (Virtual Health Library), coordinated by BIREME and composed of bibliographic databases produced by VHL network, such as LILACS, in addition to the Medline database and other types of information sources and CINAHL (Cumulative Index to Nursing and Allied Health Literature).

Results: 09 complete publications were selected to support results and discussion, only publications from 2014 to 2020 were included, in Portuguese, English and Spanish. The studies addressed the nursing care provided to women victims of domestic violence, the performance of propaedeutic techniques such as inspection, palpation and auscultation, as well as the importance of a holistic approach to quality and humanized care.

Conclusion: In this way, the forms of assistance provided by primary care nurses to women who are victims of domestic violence and the importance of carrying out the notification are identified, thus identifying the appropriate actions, adversities and possible interventions that can improve nursing care. 

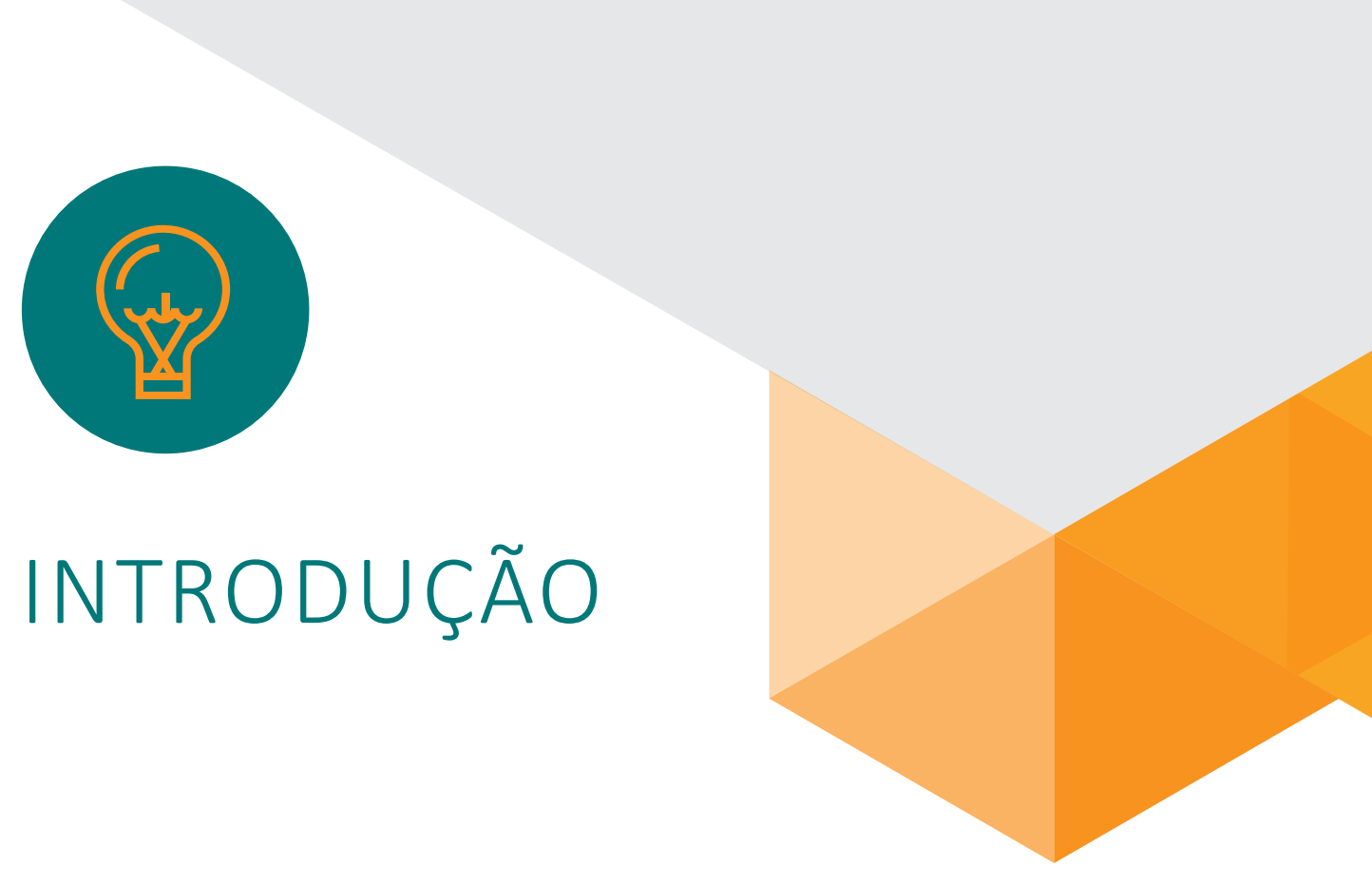

A violência doméstica contra a mulher é qualquer ação que the cause morte, danos psicológico, sexual, físico, moral ou patrimonial e que não se restringe somente ao companheiro, mais que pode advir de qualquer pessoa que esteja no convívio familiar. É um problema universal e que ocorre de forma silenciosa e dissimuladamente, independentemente de classe social e religião (OMS, 2017).

Segundo Santos et al. (2018), a violência doméstica vem sendo identificada como um grave problema de saúde pública e violação dos direitos humanos, afetando negativamente a saúde física, mental, sexual e reprodutiva das mulheres, além de aumentar a vulnerabilidade para as IST's. A baixa escolaridade, exposição à violência entre os pais, abuso durante a infância e atitudes de violência e desigualdade de gênero, são fatores contribuintes ao risco de mulheres serem vítimas de violência doméstica.

Nesse contexto, é essencial que os profissionais de enfermagem estejam capacitados para diagnosticar e prestar assistência de qualidade e de forma holística, com medidas de prevenção de agravos e reabilitação da saúde da vítima, visto que, a assistência prestada a mulher violentada na atenção primária deve ser realizado por um profissional que esteja devidamente apto para identificar e notificar o caso de agressão e encaminhar a vítima, promovendo sua autonomia e ruptura do ciclo de violência (VILLA; MACHADO, 2018).

Diante do exposto foi elaborado a seguinte questão norteadora: Como é prestada a assistência de enfermagem a mulher vítima de violência doméstica? Tendo como objetivo geral descrever através da literatura vigente a assistência de enfermagem prestada à mulher vítima de violência doméstica. E especificamente, identificar as formas de assistência de enfermagem prestada as vítimas de violência doméstica; descrever segundo a literatura a importância das notificações dos casos de violência doméstica contra a mulher; apresentar segundo a literatura ações cabíveis e possíveis soluções para aperfeiçoamento da prática de enfermagem.

Esta pesquisa justifica-se por evidenciar as estratégias da enfermagem na assistência as mulheres vítimas de violência doméstica, visto que, é um problema social e de saúde pública, pois além de afetar a saúde física, sexual e psicológica da mulher, a família também se torna vulnerável ao agressor, podendo trazer prejuízos a qualidade de vida das vítimas. 
O estudo tem como relevância abordar o papel do enfermeiro e os cuidados na assistência prestada a mulher violentada, e como o enfrentamento pode superar as limitações. Assim, os resultados desta pesquisa poderão contribuir para uma reorganização dos serviços de saúde na atenção primária enfatizando uma assistência holística à essas mulheres, identificando as condutas cabíveis, dificuldades e possíveis ações de prevenção e reabilitação da saúde das vítimas de violência doméstica.

\section{DESENVOLVIMENTO}

\section{METODOLOGIA} revisão integrativa da literatura. Este procedimento foi escolhido por possibilitar a síntese e análise do conhecimento científico já produzido sobre o tema "ANÁLISE BIBLIOGRÁFICA DA ASSISTÊNCIA DE ENFERMAGEM PRESTADA A MULHER VITTIMA DE VIOLÊNCIA DOMÉSTICA", esta revisão utilizou a metodologia proposta no estudo (OLIVEIRA et al., 2016).

A partir da questão norteadora desta revisão integrativa da literatura: "Como é realizado a assistência de enfermagem a mulher vítima de violência doméstica?" determinou a construção da estratégia PICO, que representa um acrônimo para População (P), Interesse (I), Contexto (Co), na qual foi utilizada para a localização dos estudos relevantes, que respondessem à pergunta de pesquisa, utilizou-se de descritores indexados e não indexados (palavras-chave) nos idiomas português, inglês e espanhol.

Os descritores foram obtidos a partir do Medical Subject Headings (MESH), dos Descritores em Ciências da Saúde (DeCS) e dos títulos CINAHL, como mostra o Quadro 1. Consultou-se por meio de descritores e palavras-chave as bases de dados PubMed da National Library of Medicine; BVS (Biblioteca Virtual da Saúde), coordenada pela BIREME e composta de bases de dados bibliográficas produzidas pela Rede BVS, como LILACS, além da base de dados Medline e outros tipos de fontes de informação; e CINAHL (Cumulative Index to Nursing and Allied Health Literature). 
Quadro 1. Elementos da estratégia PICO, descritores e palavras-chave utilizados. Caxias, MA, Brasil, 2020.

\begin{tabular}{|c|c|c|c|c|c|}
\hline \multicolumn{2}{|c|}{ Elementos } & Decs & Mesh & Título Cinhal & Palavras-chave \\
\hline $\mathbf{P}$ & "Paciente" & $\begin{array}{l}\text { "Patients" } \\
\text { "Pacientes" } \\
\text { "Pacientes" }\end{array}$ & "Patients" & "Patients" & $\begin{array}{l}\text { "Pacientes" } \\
\text { "Pacientes" }\end{array}$ \\
\hline I & $\begin{array}{c}\text { "Violência } \\
\text { doméstica" } \\
\text { “Assistên- } \\
\text { cia de En- } \\
\text { fermagem a } \\
\text { Mulher Víti- } \\
\text { ma de vio- } \\
\text { lência do } \\
\text { méstica" }\end{array}$ & $\begin{array}{l}\text { "Domestic Violence " } \\
\text { "Violencia Domés- } \\
\text { tica" } \\
\text { "Violencia Domés- } \\
\text { tica" } \\
\text { "Nursing Care" } \\
\text { "Atención de Enfer- } \\
\text { mería" } \\
\text { "Assistência de En- } \\
\text { fermagem" }\end{array}$ & $\begin{array}{l}\text { "Domestic } \\
\text { Violence" } \\
\text { "nursing care } \\
\text { to women } \\
\text { who are } \\
\text { victims of } \\
\text { domestic vio- } \\
\text { lence" }\end{array}$ & $\begin{array}{l}\text { "Violence Do- } \\
\text { mestic" } \\
\text { 'nursing care } \\
\text { to women who } \\
\text { are victims of } \\
\text { domestic vio- } \\
\text { lence" }\end{array}$ & $\begin{array}{l}\text { "Domestic Violence" } \\
\text { "Violence Domestic" } \\
\text { "Violência Doméstica" } \\
\text { "nursing care to women } \\
\text { who are victims of domes- } \\
\text { tic violence" } \\
\text { "Assistência de Enferma- } \\
\text { gem a Mulher Vítima de } \\
\text { violência doméstica" }\end{array}$ \\
\hline Co & $\begin{array}{c}\text { "Assistên- } \\
\text { cia Primá- } \\
\text { ria em Saú- } \\
\text { de" }\end{array}$ & $\begin{array}{l}\text { "Atención Primaria } \\
\text { de Salud" } \\
\text { "Atenção Primária } \\
\text { em Saúde" }\end{array}$ & $\begin{array}{c}\text { "Primary } \\
\text { Health Care" }\end{array}$ & $\begin{array}{c}\text { "Primary Health } \\
\text { Care" }\end{array}$ & $\begin{array}{c}\text { "Assistência Primária em } \\
\text { Saúde" }\end{array}$ \\
\hline
\end{tabular}

Fonte: Descritores, Títulos e Palavras-chaves, 2020.

Os termos utilizados durante a pesquisa foram classificados e combinados nos bancos de dados conforme o Quadro 2., resultando em estratégias específicas em cada base. 
Quadro 2. Estratégias de busca utilizadas nas bases de dados BIREME, PUBMED e CINAHL. Caxias, MA, Brasil, 2020.

\begin{tabular}{|c|c|c|c|c|}
\hline BASE DE DADOS & ESTRATÉGIA DE BUSCA & 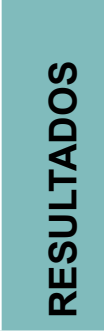 & 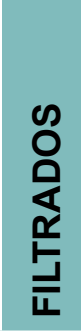 & 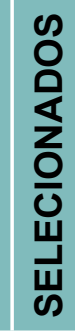 \\
\hline $\begin{array}{c}\text { BIREME } \\
\text { (descritores Decs) }\end{array}$ & $\begin{array}{l}\text { (tw:(violência doméstica contra a mulher)) AND (tw:(Assis- } \\
\text { tência de enfermagem )) }\end{array}$ & 107 & 16 & 9 \\
\hline $\begin{array}{c}\text { PubMed } \\
\text { (descriptors MeSH) }\end{array}$ & $\begin{array}{l}\text { violencia domestica AND ("last } 5 \text { years"[PDat] AND Humans[- } \\
\text { Mesh]) partner[All Fields] AND ("violence"[MeSH Terms] OR } \\
\text { "violence"[All Fields]) AND ("diagnosis"[Subheading] OR "diagno- } \\
\text { sis"[All Fields] OR "screening"[All Fields] OR "mass screening"[- } \\
\text { MeSH Terms] OR ("mass"[All Fields] AND "screening"[All Fields]) } \\
\text { OR "mass screening"[All Fields] OR "screening"[All Fields] OR } \\
\text { "early detection of cancer"[MeSH Terms] OR ("early"[All Fields] } \\
\text { AND "detection"[All Fields] AND "cancer"[All Fields]) OR "early } \\
\text { detection of cancer"[All Fields]) ("domestic violence"[MeSH Ter- } \\
\text { ms] OR ("domestic"[All Fields] AND "violence"[All Fields]) OR } \\
\text { "domestic violence"[All Fields]) AND ("women"[MeSH Terms] OR } \\
\text { "women"[All Fields]) }\end{array}$ & 2638 & 110 & 3 \\
\hline $\begin{array}{l}\text { CINAHL (CINAHL } \\
\text { Headings) }\end{array}$ & Patients AND (domestic violence) AND (nursing care) & 23 & 15 & 0 \\
\hline
\end{tabular}

Fonte: Bases de dados, 2020.

Como critérios de inclusão utilizaram-se estudos disponíveis em sua totalidade, publicados nos últimos seis anos, de 2014 a 2020, nos idiomas Português, Espanhol e Inglês. Foram excluídos da busca inicial capítulos de livros, resumos, textos incompletos, teses, dissertações, relatos técnicos e outras formas de publicações que não são artigos científicos completos.

\section{RESULTADOS}

Os estudos foram classificados e organizados conforme a Tabela 01. Ao todo nove estudos incluídos nesta revisão, cinco estavam na língua inglesa $(55,5 \%)$. A maioria das publicações foram concentradas no ano de 2017 e 2018 (75\%), com quatro estudos de abordagem qualitativa (66,6\%), e apenas um de abordagem quantitativa (33,3\%).

O nível de evidência predominante foi considerado médio, composto por estudo de caso (11,1\%), estudo transversal (22,2\%), ensaio clínico randomizado (11,1\%), estudo de coorte $(11,1 \%)$ e revisão sistemática $(44,4 \%)$; e todos os estudos (100\%) obtiveram grau de recomendação " $A$ " para mudança na prática clínica.

Do total de estudos analisados 55,5\% originados no Brasil e 33,3\% EUA e 11,1\% na Espanha. Os artigos selecionados foram divulgados em nove periódicos diferentes, sendo cinco nacionais $(55,5 \%)$ e quatro internacionais $(44,4 \%)$. 
Tabela 1. Análise descritiva das produções científicas acerca da análise bibliográfica da assistência de enfermagem a mulher vítima de violência doméstica. Caxias, MA, Brasil, 2020.

\begin{tabular}{|c|c|c|}
\hline VARIÁVEIS & $\mathbf{N}$ & $\%$ \\
\hline \multicolumn{3}{|l|}{ Abordagem do estudo } \\
\hline Quantitativo & 03 & 33,3 \\
\hline Qualitativo & 06 & 66,6 \\
\hline \multicolumn{3}{|l|}{ Delineamento da pesquisa } \\
\hline Estudo de caso & 01 & 11,1 \\
\hline Estudo transversal & 02 & 22,2 \\
\hline Ensaio clinico Randomizado & 01 & 11,1 \\
\hline Estudo de Coorte & 01 & 11,1 \\
\hline Revisão Sistemática & 04 & 44,4 \\
\hline \multicolumn{3}{|l|}{ Idioma } \\
\hline Inglês & 05 & 55,5 \\
\hline Espanhol & 03 & 33,3 \\
\hline Português & 01 & 11,1 \\
\hline \multicolumn{3}{|l|}{ Classificação de evidência } \\
\hline Um & 04 & 44,4 \\
\hline Três & 02 & 22,2 \\
\hline Quatro & 03 & 33,3 \\
\hline \multicolumn{3}{|l|}{ Grau de recomendação } \\
\hline$A$ & 09 & 100 \\
\hline \multicolumn{3}{|l|}{ Procedência } \\
\hline Espanha & 01 & 11,1 \\
\hline EUA & 03 & 33,3 \\
\hline Brasil & 05 & 55,5 \\
\hline
\end{tabular}

Fonte: Elaborado pelos autores, 2020.

Os estudos avaliaram a assistência de enfermagem prestada a mulher vítima de violência doméstica conforme pode ser observado no Quadro 03. Foram apresentadas as adversidades que surgem durante a assistência realizada pelo enfermeiro, as condições de trabalho dos profissionais, a necessidade da educação continuada afim de adquirir novos conhecimentos relacionados ao atendimento à mulher violentada, a falta de recursos que possa promover saúde, a importância da realização da notificação do caso de violência, tratamento e encaminhamento da vítima. Como também, identificou a necessidade de reorganização dos serviços de saúde na atenção primária, que são fatores essenciais para uma assistência de qualidade e humanizada. 
Quadro 3. Publicações incluídas segundo o título do artigo, autor, objetivo principal e perfil amostral. Caxias, MA, Brasil, 2020.

\begin{tabular}{|c|c|c|c|c|}
\hline $\begin{array}{l}\text { ORDEM } \\
\text { E BASE }\end{array}$ & TÍTULO DO ARTIGO & AUTORES & OBJETIVO PRINCIPAL & PERFIL AMOSTRAL \\
\hline $\begin{array}{c}1 \\
\text { BIREME }\end{array}$ & $\begin{array}{l}\text { Violence against } \\
\text { women in the single } \\
\text { health system. }\end{array}$ & $\begin{array}{l}\text { CAVALCANTI } \\
\text { et al. (2020) }\end{array}$ & $\begin{array}{l}\text { Caracterizar a produção científica } \\
\text { acerca da violência contra mulher } \\
\text { e suas repercussões sociais, em } \\
\text { periódicos online no âmbito da } \\
\text { saúde, publicados no período de } \\
2011 \text { a } 2016 \text {. }\end{array}$ & \\
\hline $\begin{array}{c}2 \\
\text { BIREME }\end{array}$ & $\begin{array}{l}\text { Assimilação teórica } \\
\text { e prática da violên- } \\
\text { cia doméstica: pro- } \\
\text { fissionais de enfer- } \\
\text { magem atendendo } \\
\text { vítimas na atenção } \\
\text { primária. }\end{array}$ & $\begin{array}{l}\text { AMARIJO et } \\
\text { al. (2018) }\end{array}$ & $\begin{array}{l}\text { Analisar a assimilação teórica e } \\
\text { prática da violência doméstica } \\
\text { contra a mulher (VDCM) entre pro- } \\
\text { fissionais de enfermagem, conside- } \\
\text { rando o atendimento às vítimas na } \\
\text { unidade de saúde da família }\end{array}$ & $\begin{array}{l}\text { Foram convidadas } \\
\text { a participar do } \\
\text { estudo todas as } \\
\text { enfermeiras e téc- } \\
\text { nicas de enferma- } \\
\text { gem das unidades }\end{array}$ \\
\hline $\begin{array}{c}3 \\
\text { BIREME }\end{array}$ & $\begin{array}{l}\text { Assistência de } \\
\text { enfermagem às } \\
\text { mulheres vítimas } \\
\text { de violência domés- } \\
\text { tica. }\end{array}$ & $\begin{array}{l}\text { LIMA et al. } \\
\text { (2017) }\end{array}$ & $\begin{array}{l}\text { Identificar na literatura ações de- } \\
\text { senvolvidas por enfermeiros da } \\
\text { Estratégia de Saúde da família com } \\
\text { vítimas de violência doméstica. }\end{array}$ & \\
\hline $\begin{array}{c}4 \\
\text { PUB- } \\
\text { MED }\end{array}$ & $\begin{array}{l}\text { Nurse education } \\
\text { and understanding } \\
\text { related to domestic } \\
\text { violence and abuse } \\
\text { against women: An } \\
\text { integrative review of } \\
\text { the literature. }\end{array}$ & $\begin{array}{l}\text { ALSHAMMAR; } \\
\text { MCGARRY; } \\
\text { HIGGINBOT- } \\
\text { TOM, (2018) }\end{array}$ & $\begin{array}{l}\text { Explorar a literatura anterior re- } \\
\text { lacionada ao entendimento dos } \\
\text { enfermeiros sobre violência por } \\
\text { parceiro íntimo ou violência e abu- } \\
\text { so doméstico contra mulheres e as } \\
\text { lacunas no ensino de enfermagem, } \\
\text { a fim de usar os resultados como } \\
\text { linha de base para informar estra- } \\
\text { tégias potenciais de intervenção e } \\
\text { desenvolvimento curricular. }\end{array}$ & \\
\hline $\begin{array}{l}5 \\
\text { PUB- } \\
\text { MED }\end{array}$ & $\begin{array}{l}\text { The role of attitudes } \\
\text { to, and the frequen- } \\
\text { cy of, domestic } \\
\text { violence encounters } \\
\text { in the healthcare } \\
\text { professionals' han- } \\
\text { dling of domestic } \\
\text { violence cases. }\end{array}$ & $\begin{array}{l}\text { ZORJAN; } \\
\text { SMRKE; } \\
\text { ŠPRAH, } \\
\text { (2017) }\end{array}$ & $\begin{array}{l}\text { Examinar a relação entre as atitu- } \\
\text { des dos profissionais de saúde em } \\
\text { relação à aceitabilidade da vio- } \\
\text { lência doméstica e suas respostas } \\
\text { ao lidar com vítimas de violência } \\
\text { doméstica. }\end{array}$ & $\begin{array}{l}322 \text { profissionais } \\
\text { de saúde (mé- } \\
\text { dicos, dentistas, } \\
\text { equipe de enfer- } \\
\text { magem e outros } \\
\text { profissionais de } \\
\text { saúde; } 85,2 \% \text { do } \\
\text { sexo feminino). }\end{array}$ \\
\hline
\end{tabular}




\begin{tabular}{|c|c|c|c|c|}
\hline $\begin{array}{c}6 \\
\text { BIREME }\end{array}$ & $\begin{array}{l}\text { Mulheres em situa- } \\
\text { ção de violência: } \\
\text { (re) pensando a } \\
\text { escuta, Vínculo e } \\
\text { visita. }\end{array}$ & $\begin{array}{l}\text { HEISLER et al. } \\
\text { (2018) }\end{array}$ & $\begin{array}{l}\text { Relatar a experiência de ações } \\
\text { educativas de uma pesquisa parti- } \\
\text { cipante com profissionais da Estra- } \\
\text { tégia Saúde da Família. }\end{array}$ & $\begin{array}{l}38 \text { profissionais } \\
\text { de saúde inte- } \\
\text { grantes de seis } \\
\text { equipes de ESF } \\
\text { de um município } \\
\text { da região noroes- } \\
\text { te do estado do } \\
\text { Rio Grande do } \\
\text { Sul, Brasil, a fim } \\
\text { de (re) pensar a } \\
\text { escuta, o vínculo } \\
\text { e a visita domici- } \\
\text { liar às mulheres } \\
\text { em situação de } \\
\text { violência. }\end{array}$ \\
\hline $\begin{array}{l}7 \\
\text { PUB- } \\
\text { MED }\end{array}$ & $\begin{array}{l}\text { Digital Education of } \\
\text { Health Professio- } \\
\text { nals on the Mana- } \\
\text { gement of Domestic } \\
\text { Violence: Syste- } \\
\text { matic Review and } \\
\text { Meta-Analysis by } \\
\text { the Digital Health } \\
\text { Education Collabo- } \\
\text { ration. }\end{array}$ & $\begin{array}{l}\text { DIVAKAR et } \\
\text { al. (2019) }\end{array}$ & $\begin{array}{l}\text { Avaliar a efetividade da educação } \\
\text { digital das profissões da saúde no } \\
\text { ambiente doméstico. violência } \\
\text { comparada com a das formas tra- } \\
\text { dicionais ou sem intervenção. }\end{array}$ & $\begin{array}{l}\text { Seis estudos com } \\
631 \text { participantes } \\
\text { preencheram os } \\
\text { critérios de inclu- } \\
\text { são. }\end{array}$ \\
\hline $\begin{array}{c}8 \\
\text { BIREME }\end{array}$ & $\begin{array}{l}\text { Aspectos éticos e } \\
\text { legais no cuidado } \\
\text { de enfermagem às } \\
\text { vítimas de violência } \\
\text { doméstica. }\end{array}$ & $\begin{array}{l}\text { ACOSTA et } \\
\text { al. (2017) }\end{array}$ & $\begin{array}{l}\text { Analisar o conhecimento de en- } \\
\text { fermeiras hospitalares sobre os } \\
\text { aspectos éticos e legais no cui- } \\
\text { dado de enfermagem às vítimas } \\
\text { de violência doméstica }\end{array}$ & $\begin{array}{l}\text { Três enfermeiras, } \\
\text { com tempo míni- } \\
\text { mo de dois meses } \\
\text { de atuação no } \\
\text { referido setor, por } \\
\text { se considerar que } \\
\text { com esse tempo } \\
\text { as informantes } \\
\text { tivessem tido a } \\
\text { oportunidade de } \\
\text { prestar cuidados } \\
\text { a vítimas de vio- } \\
\text { lência. }\end{array}$ \\
\hline $\begin{array}{c}9 \\
\text { SciELO }\end{array}$ & $\begin{array}{l}\text { Why do certain } \\
\text { primary health care } \\
\text { teams respond bet- } \\
\text { ter to intimate part- } \\
\text { ner violence than } \\
\text { others? }\end{array}$ & $\begin{array}{l}\text { GOICOLEA et } \\
\text { al. (2019) }\end{array}$ & $\begin{array}{l}\text { Analisar como as condições no ní- } \\
\text { vel da equipe influenciaram as res- } \\
\text { postas dos profissionais de saúde a } \\
\text { violência por parceiro íntimo. }\end{array}$ & $\begin{array}{l}\text { Quatro equipes de } \\
\text { atenção primária à } \\
\text { saúde localizadas } \\
\text { em uma região no } \\
\text { sul da Espanha. }\end{array}$ \\
\hline
\end{tabular}

Fonte: Elaborado pelos autores, 2020. 
Figura 1. Fluxograma de protocolo de intervenções de enfermagem para a realização da assistência em mulheres vítimas de violência doméstica. Caxias, MA, Brasil, 2020.

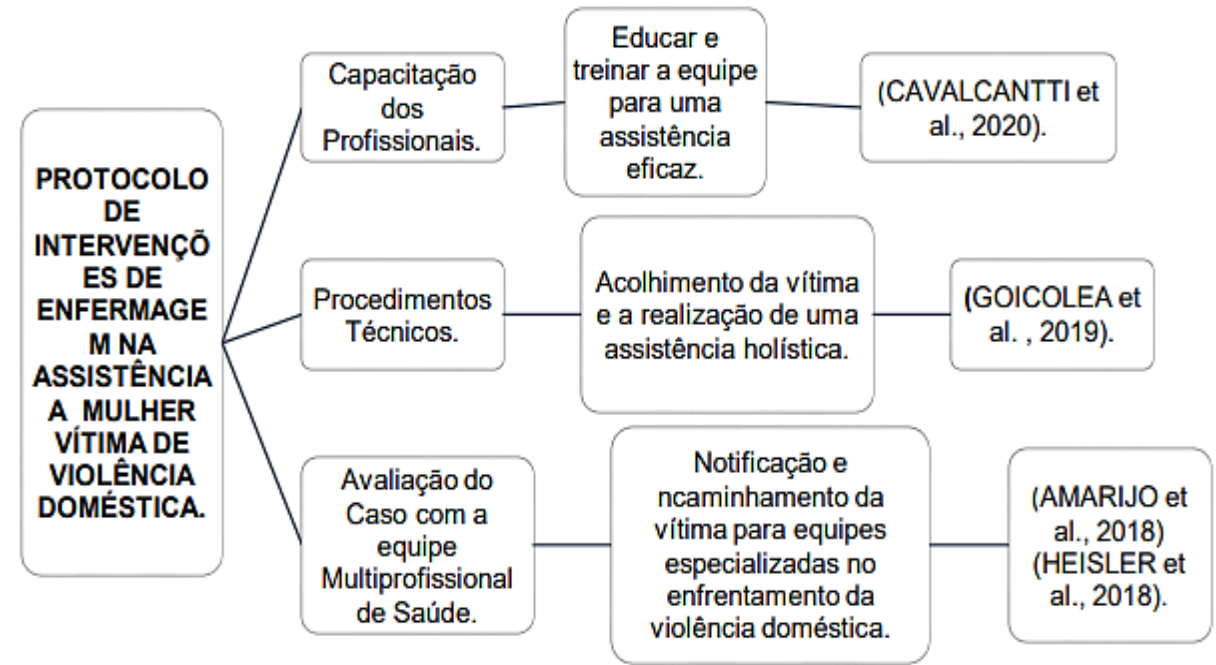

Fonte: Elaboração autoral, 2020.

Os artigos selecionados, explanam sobre de que forma a enfermagem pode realizar assistência a mulher vítima de violência doméstica evidenciando o acolhimento como conduta primordial para uma assistência de qualidade e a necessidade de humanização no atendimento, e a limitação do conhecimento dos profissionais de enfermagem em relação ao atendimento a mulheres violentadas (ZORJAN; SMRKE; ŠPRAH, 2017; ACOSTA et al., 2017; LIMA et al ., 2017; AMARIJO et al., 2018; HEISLER et al., 2018; GOICOLEA et al., 2019; CAVALCANTTI et al., 2020; DIVAKAR et al., 2019; ALSHAMMARI; MCGARRY; HIGGINBOTTOM, 2018).

Diante do exposto Acosta et al. (2017); Amarijo et al. e Heisler et al. (2018) expõem que os profissionais de saúde relatam despreparo no que se refere as competências legais no atendimento às vítimas de violência que vai desde o desconhecimento da obrigatoriedade da notificação compulsória do caso de violência, avaliação do caso com a equipe multiprofissional de saúde ao encaminhamento da vítima.

Em relação as vítimas segundo a literatura independem de cor, raça, religião visto que, a violência ocorre em apenas razão do sexo. Nos estudos de Divakar et al. (2019) afirmam que as vítimas são mulheres em idade reprodutiva entre 15 a 49 anos e em razão da insegurança a vítima opta por não expor a ocorrência dos fatos ao profissional de saúde durante a realização da assistência.

A assistência de enfermagem a mulher vítima de violência doméstica pode ser aperfeiçoada desenvolvida de forma que possa contribuir significativamente na prevenção do feminicídio, visto que, é um problema de saúde mundial que requer intervenções imediatas e eficazes como: capacitação dos profissionais, apoio emocional a vítima, habilidade de comunicação e investimento na promoção de saúde e prevenção de agravos nas unidades básica de saúde (UBS) (ALSHAMMARI; MCGARRY; HIGGINBOTTOM, 2018; DIVAKAR et al., 2019).

\section{DISCUSSÃO}

A princípio, faz-se necessário a capacitação dos profissionais de enfermagem com a especialização no conhecimento sobre a assistência de enfermagem prestada as mulheres vítimas de violência doméstica, permitindo que novas ações de apoio social sejam desenvolvidas. Ressalta-se a necessidade 
da implementação e fortalecimento de políticas públicas para uma efetiva conscientização sobre a importância da denúncia, bem como a disponibilidade de informações sobre os serviços de atendimento disponíveis às vítimas (CAVALCANTTI et al., 2020).

No estudo de Goicolea et al. (2019) destacam que o acolhimento a vítima contribui significativamente no tratamento, visto que, a insegurança das mulheres ao procurar os serviços de saúde pode estar relacionada a maneira de como é abordagem do profissional de saúde, sendo assim necessário a adoção de uma abordagem holística, relacionando as queixas inespecíficas do paciente às circunstâncias sociais e não tendo como prioridade apenas a prescrição de medicamentos para tratar os sintomas. A assistência prestada a mulher vítima de violência não se restringe apenas em anamnese e exame físico, mas deve ser articulada com outros setores específicos na área, para assim promover segurança, acolhimento e respeito.

Desta forma Amarijo et al. (2018) descrevem os cuidados de enfermagem como a abordagem holística do paciente, identificação de achados que caracterizam a violência, a realização de procedimentos técnicos, notificação e encaminhamento da vítima, visto que, as vítimas devem ser orientadas sobre seus direitos e os recursos que poderão utilizar no enfrentamento da violência.

A notificação dos casos de violência contra a mulher pode ser definida como uma intervenção norteadora do sistema único de saúde (SUS), pois permite a diferenciação dos tipos de violência, caracterização das vítimas e agressores, além da padronização e sistematização de informações com a finalidade de identificar o perfil das vítimas e definição do grau de parentesco ou relação entre agressores e vítimas, além de instituir tratamento e elaborar novas ações de intervenção que possa minimizar os casos de violência (CAVALCANTTI et al., 2020).

Apesar da importância da notificação para fins epidemiológicos na construção de perfis no Sistema de informação de agravos de notificação (SINAN), em um estudo descritivo realizado nos hospitais que tratam casos de violência no Rio Grande do Sul- RS, divergem, afirmando desconhecimento e/ou despreparo dos profissionais de enfermagem acerca das condutas nos casos de violência doméstica, pois equivocavam as fichas de notificação e encaminhamento da vítima, associando-a denúncia policial. (ACOSTA et al., 2017).

Nos estudos feitos por Ribeiro e Silva (2018) enfatizam que a omissão da notificação compulsória na atenção primária, consequentemente pode ocultar os casos de violência, visto que, é obrigatório o preenchimento da ficha de notificação. Sobretudo, essa intervenção é de grande relevância no processo de trabalho da enfermagem e da equipe multiprofissional de saúde. A notificação compulsória dos casos de violência contra a mulher, pode ser um instrumento eficaz para implementação de políticas públicas as vítimas de violência da comunidade.

Assim a Estratégia Saúde da Família é responsável pela reorganização do processo de trabalho, visando o enfrentamento das limitações existentes na assistência de enfermagem as mulheres vítimas de violência doméstica. Nesse sentido é de competência da enfermagem o desenvolvimento do relacionamento interpessoal enfermeiro/paciente, acolhimento da vítima, e identificação da violência através da inspeção, palpação e ausculta da paciente, tendo em vista a identificação de alterações do estado de saúde da vítima, contribuindo para a formação do diagnóstico (SIMÕES et al. 2019).

A enfermagem pode intervir de forma articulada com outros setores, com ações de educação em saúde através da realização de campanhas de prevenção da violência contra a mulher no âmbito escolar e sociedade em geral contribuindo para que a vítima reconheça o ato de violência e consequentemente possa realizar a denúncia. Embora, exista a complexidade de implementação de intervenções, é necessário a criação de uma rede assistencial em prol da saúde da mulher e apoio às vítimas (SANTOS et al., 2019). 


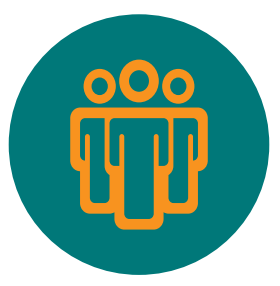

\section{CONCLUSÃO}

O estudo permitiu a identificação das limitações existentes na assistência de enfermagem nos casos de violência doméstica contra a mulher, desencadeadas pela falta de capacitação dos profissionais, falha na realização das notificações, falta de recursos para continuidade dos serviços nas unidades básica de saúde, condições de trabalho desfavoráveis para realização da promoção da saúde da mulher, prevenção de agravos e reabilitação da saúde da vítima.

É necessário que o enfermeiro esteja capacitado para lidar com essas adversidades no âmbito da atenção primária, visto que, se localiza dentro da comunidade e é responsável por resolver grande parte das demandas da população. É necessária uma abordagem holística correlacionando as queixas inespecíficas da paciente e os achados anormais durante a consulta de enfermagem.

Almeja-se que esse estudo possa contribuir significativamente no campo do saber para os profissionais de saúde e na implantação de protocolos de atendimento as mulheres violentadas, com a criação de ações que possa melhorar a assistência de enfermagem prestada as mulheres vítimas de violência doméstica, onde os gestores possam implantar intervenções com o foco na saúde da mulher e melhorias das condições de trabalho, e automaticamente na melhora da assistência realizada visto que, os profissionais de enfermagem sintam-se inseguro para tomar as condutas cabíveis diante dessa adversidade. 


\section{REFERÊNCIAS}

ACOSTA, D. F. et al. Aspectos éticos e legais no cuidado de enfermagem às vítimas de violência doméstica. Texto contexto - enferm., Florianópolis, v. 26, n. 3, e6770015, 2017. Disponível em: <http:// www.scielo.br/scielo.php?script=sci_arttext\&pid=S0104-07072017000300311\&lng=en\&nrm=iso>. Acesso em: 29 nov. 2019.

ALSHAMMARI, K. F.; MCGARRY, J.; HIGGINBOTTOM, G. M. A. Nurse education and understanding related to domestic violence and abuse against women: an integrative review of the literature. Nursing Open, [S.I.], v. 5, n. 3, p. 237-253, 12 mar. 2018. Disponível em: <https://pubmed.ncbi.nIm.nih. gov/30062016/>. Acesso em: 29 nov. 2019.

AMARIJO, C. L. et al. Assimilação teórica e prática da violência doméstica: profissionais de enfermagem atendendo vítimas na atenção primária. Revista Enfermagem Uerj, [S.I.], v. 26, e33874, nov. 2018. Disponível em: <https://www.e-publicacoes.uerj.br/index.php/enfermagemuerj/article/view/33874>. Acesso em: 29 nov. 2019.

CAVALCANTI, G. M. B. et al. Violence against women in the single health system. Revista de Pesquisa Cuidado É Fundamental Online, [S.I.], v. 12, p. 146-154, 10 jan. 2020. Disponível em: <http://seer. unirio.br/index.php/cuidadofundamental/article/view/7148/pdf>. Acesso em: 29 nov. 2019.

DIVAKAR, U. et al. Digital Education of Health Professionals on the Management of Domestic Violence: systematic review and meta-analysis by the digital health education collaboration. Journal of Medical Internet Research, [S.I.], v. 21, n. 5, e13868, 23 maio 2019. Disponível em: <https://www.jmir. org/2019/5/e13868/>. Acesso em: 29 nov. 2019.

ERCOLE, Flávia Falci; MELO, Laís Samara de; ALCOFORADO, Carla Lúcia Goulart Constant. Integrative review versus systematic review. Revista Mineira de Enfermagem, [S.I.], v. 18, n. 1, p. 9-11, 2014. Disponível em: < https://cdn.publisher.gn1.link/reme.org.br/pdf/v18n1a01.pdf>.

GOICOLEA, Isabel et al. Why do certain primary health care teams respond better to intimate partner violence than others?. A multiple case study. Gac Sanit, Barcelona, v. 33, n. 2, p. 169-176, abr. 2019. Disponível em: <http://scielo.isciii.es/scielo.php?script=sci_arttext\&pid=S0213-91112019000200169\&lng=en\&nrm=iso>. Acesso em: 29 nov. 2019.

HEISLER, E. D. et al. Mulheres em situação de violência: (re) pensando a escuta, vínculo e visita. Revista de Enfermagem Ufpe On Line, [S.I.], v. 12, n. 1, p. 265-272, 1 jan. 2018. Disponível em: <https://periodicos.ufpe.br/revistas/revistaenfermagem/article/view/230504/26102>. Acesso em: 29 nov. 2019.

LIMA, L. A. A. et al. Nursing care for women victims of domestic violence / Assistência de enfermagem às mulheres vítimas de violência doméstica / Los cuidados de enfermería para las mujeres víctimas de la violencia doméstica. Revista de Enfermagem da Ufpi, [S.I.], v. 6, n. 2, p. 65-68, 1 jun. 2017. Disponível em: <https://ojs. ufpi.br/index.php/reufpi/article/view/5783/pdf>. Acesso em: 29 nov. 2019.

OLIVEIRA, F. B. M. et al. Relação entre a sobrecarga de trabalho e erros de administração de medicação na assistência hospitalar. Revista Ciências \& Saberes, Caxias, v.2, n.2. p. 325-334, out./dez.2016. Disponível em: < https://www.facema.edu.br/ojs/index.php/ReOnFacema/article/view/212/81>. Acesso em: 23 abr. 2020. 
ORGANIZAÇÃO MUNDIAL DA SAÚDE (OMS). (Org.). Folha informativa- Violência contra as mulheres. Geneva: OMS, 2017. Disponível em: <https://www.paho.org/bra/index.php?option=com_content\&view=article\&id=5669:folha-informativa-violencia-contra-as-mulheres\&ltemid=820>. Acesso: 29 abr. 2019.

RIBEIRO, R. U. P.; SILVA, A. L. Notificação compulsória de violência na atenção básica à saúde: o que dizem os profissionais? Revista LEVS, Marília, p.115-30, mai. 2018.

SANTOS, D. S. et al. Violência doméstica contra a mulher: visão de enfermeiros pós-graduandos em obstetrícia no Piauí. J. nurs. health., [S.I.], v. 9, n. 3, e199310, 2019. Disponível em: < https://periodicos.ufpel.edu.br/ojs2/index.php/enfermagem/article/view/17198/10823>. Acesso em: 29 abr. 2019.

SIMOES, A. V. et al. Identificação e conduta da violência doméstica contra a mulher sob a ótica dos estudantes universitários. Enfermería Actual de Costa Rica, San José, n. 37, p. 95-109, dez. 2019. Disponível em: <http://www.scielo.sa.cr/scielo.php?script=sci_arttext\&pid=S1409-45682019000200095\&lng=en\&nrm=iso>. Acesso em: 29 nov. 2020.

VILLA, E. N. R. M.; MACHADO, B. A. O mapa do feminicídio na polícia civil do Piauí: uma análise organizacional-sistêmica. Revista Opinião Jurídica, Fortaleza, [S.I.], v. 16, n. 22, p. 86-107, 23 mar. 2018. Disponível em: < https://periodicos.unichristus.edu.br/opiniaojuridica/article/view/1478/621>. Acesso em: 29 abr. 2019.

ZORJAN, S.; SMRKE, U.; ŠPRAH, L. The role of attitudes to, and the frequency of, domestic violence encounters in the healthcare professionals' handling of domestic violence cases. Slovenian Journal Of Public Health, [S.I.], v. 56, n. 3, p. 166-171, 26 set. 2017. Disponível em: <https://www.ncbi.nlm.nih. gov/pmc/articles/PMC5504542/>. Acesso em: 29 nov. 2019. 